\title{
Learning-based Event Response for Marine Robotics
}

\author{
Matthew Bernstein ${ }^{1} \quad$ Rishi Graham ${ }^{2} \quad$ Danelle Cline $^{2} \quad$ John M. Dolan ${ }^{1} \quad$ Kanna Rajan $^{2}$
}

\begin{abstract}
Robotic vehicles have become a critical tool for studying the under-sampled coastal ocean. This has led to new paradigms in scientific discovery. The combination of agility, reactivity, and persistent presence makes autonomous robots ideal for targeted sampling of elusive, episodic events such as algal blooms. In order to achieve this goal, they need to be deployed at the right place and time. To that end, we have designed and will soon deploy a shore-based event recognition technology to continuously monitor remote sensing imagery for algal blooms as targets for robotic field experiments. A Support Vector Machine underlies a field-tested decision support system which scientists will consult prior to deploying robots in the coastal ocean. Our aim is to target oceanographic field experiments for evaluation and verification.
\end{abstract}

\section{INTRODUCTION}

The coastal ocean plays an important social and economic role and is critical to understanding the impact of a changing climate. Anthropogenic input over years has created substantial perturbation, leading to dramatic swings in organism abundance and community structure. Algae blooms are frequent visible signs of these changes. Some generate toxins which have a substantial impact on the human food-chain. [1], e.g., estimate significant economic impact resulting from such Harmful Algal Blooms (HABs). Despite years of study, bloom initiation and life-cycle are still subjects of considerable debate within the ocean science community.

Our effort applies Machine Learning (ML) techniques pioneered by the computational and robotic sciences to detecting and predicting phenomena in the coastal ocean from heterogenous data sources including expert knowledge. This will allow us to rapidly reposition our autonomous underwater vehicles (AUVs). These vehicles come with a rich suite of advanced sensors required to resolve interacting physical, chemical, biological, and geological phenomena. With mission durations of up to 22 hours, they measure physical properties continuously and can acquire water samples for laboratory analysis within dynamic features such as blooms. However, apriori surveys require robotic assets to cover mesoscale $\left(>50 \mathrm{~km}^{2}\right)$ regions repeatedly for weeks. Targeted sampling augmented by prior models yields significant cost savings. Typical oceanographic model performance is particularly poor at small scales and in the biogeochemically diverse coastal environments, making the questions of where and when to sample critical.

\footnotetext{
${ }^{1} \mathrm{M}$. Bernstein and J. Dolan are with the Robotics Institute, Carnegie Mellon University mbernstedandrew. cmu.edu and jmdecs. cmu.edu

2 R. Graham, D. Cline, and K. Rajan are with the Monterey Bay Aquarium Research Institute \{rishi, dcline, kanna.rajan\}embari.org
}

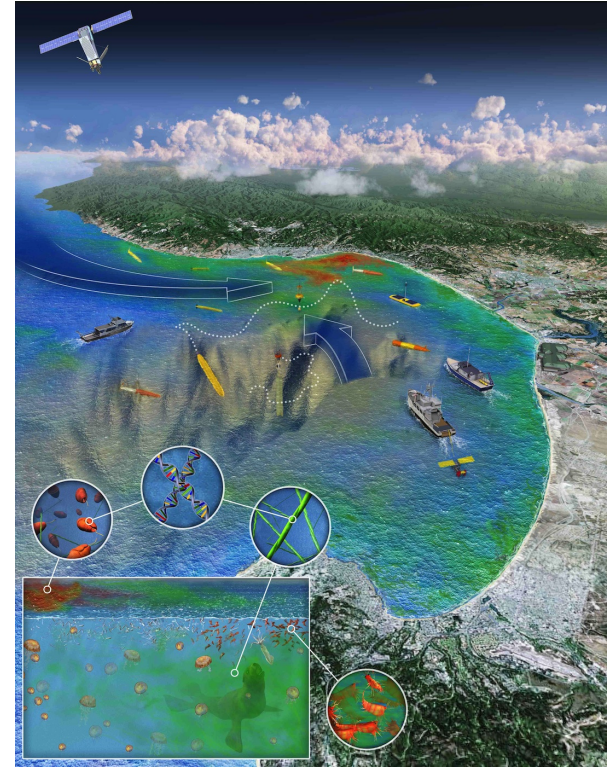

Fig. 1: Illustrative use of multiple robots and vessels sampling blooms in Monterey Bay as part of CANON. Remote sensing data, ocean models, and prior knowledge inform the activity of a heterogeneous network of mobile assets in the water.

Our work centers around field experiments with multiple AUVs in the water aided by the Oceanographic Decision Support System (ODSS) on shore or ship [2]. The ODSS is a software tool ${ }^{1}$ designed to enable situational awareness, data visualization, collaborative information sharing and planning for the Controlled Agile and Novel Observation Network (CANON) program ${ }^{2}$ [3], [4]. Our Dorado-class AUV in particular has two important features which make it suitable for targeted HAB sampling. First is the Gulper water-sampler [5], consisting of ten 2-liter syringes in the midsection of the vehicle. Second, it has an advanced control system which synthesizes abstract plans from shore-based commands, is responsive to failure using in situ plan repair and recovery, and can use a diverse set of sampling techniques which can be dynamically modified from shore [6], [7]. Cued by data-driven models in the ODSS, scientists can alter where, when and how the AUV will take samples.

In this paper we show how such an informative model can be obtained for detecting and predicting coastal ocean phenomena using various data sources and expert knowledge. We focus on algal blooms, and automatic detection from visible coloration in remote sensing data. Such blooms may or may not be harmful, but scientists

\footnotetext{
${ }^{1}$ http://odss.mbari.org

${ }^{2}$ http://www.mbari.org/canon/
} 
can respond to the event trigger in the ODSS with robotic surveys designed to return water samples for further analysis. Using statistical techniques from ML, we show how such models can be built.

The novelty of this work is two-fold. First, we bring together ML methods rarely used in oceanography or in collaborative field experiments driven by systematic computational methods such as those behind the ODSS. Second, we do so in the context of actual field experiments in an inter-disciplinary environment with scientists, engineers and operations personnel out at sea. Fig. 1, illustrates such experimentation with bloom formation in the northern Monterey Bay incubator area; an upcoming March 2013 field experiment in the Southern California Bight is expected to validate this approach.

This paper is organized as follows. Section II summarizes related work in the fields of ocean science and robotic exploration, while Section III places this effort within the context of the ODSS. Section IV gives brief descriptions of some of the ML tools used in this paper. Section V discusses the remotely sensed data products used in our approach, and some of the preprocessing steps. Section VI presents results and analysis, with conclusions and future directions in Section VII.

\section{RELATED WORK}

Significant prior work, some of it in our coastal domain of Monterey Bay, has used remote sensing images to manually identify blooms. [8] found insight into the expansion, retention, and dispersion of blooms in Seaviewing Wide Field-of-view Sensor (SeaWiFS) imagery. [9] followed a persistent bloom through changing winds, providing an interesting test case to motivate using automation and ML for future similar studies. In these works, preliminary event detection from remote sensing sources was followed up by targeted water sampling by an AUV.

More recently, [10] documented the detection and advection of blooms using satellite imagery and surface radar data and marked the initial use of the ODSS with elements of machine learning. [11] used a binary classification of chlorophyll data to validate a dynamic model of algal bloom evolution in the North Sea.

Correspondingly there have been studies using ML to enhance oceanographic experiments. [12] presents a Support Vector Machine (SVM) based method of classifying images by their color histograms. While they focus on large sets of images and indexed storage and retrieval, we concentrate on multivariate pixel-wise classification. In [13] a method is developed for using multiple remote sensing variables to detect HABs based on linear discriminant analysis, while [14] uses ocean color from SeaWiFS to detect chlorophyll anomalies, providing an early warning system for blooms of a particular toxic algae in the eastern Gulf of Mexico. The focus in these efforts is on direct detection of specific species manually, with ground truth coming from a small set of labels generated from in situ data. Our goal, instead, is to predict one feature from remote sensing data automatically. [15] uses SVM regression for predicting chlorophyll from reflectance data. We, however, focus on classification rather than regression, incorporating multiple features and spatial filters.

Robotic response to environmentally triggered events has long been a focus of the robotics community. [16] describes how forest fires, initially detected from remote sensing data or other means, may be closely monitored by Unmanned Air Vehicles. [17] describes a global sensor network comprised of low-resolution event detection followed up by targeted high-resolution imaging. [18] presents a pollution cleanup example, and [19] provides a comprehensive overview of robotic environmental monitoring. In this paper, we focus on refinement of the environmental event detection mechanism for triggering robotic response.

\section{An Architecture for EVEnt Response}

The ODSS provides a portal with which oceanographers can design, test and evaluate hypotheses with at-sea experimentation using mobile robots [3], [2]. Another goal is to couple human decision-making with probabilistic modeling and learning to enable environmental field model discovery and refinement. Machine learning algorithms will run automatically and alert the user of an event of interest. Our objective is to focus on bloom discovery to inform scientists of any significant event of potential scientific interest for further study, elucidation, and response.

We envision the use of an event detection toolbox for CANON during an initiated field experiment based on a model of blooms statistically learned from remote sensing images. The model is part of an ODSS backend with a situational awareness tool the scientist can consult at the front-end. In a typical scenario, in situ data arriving from multiple mobile or stationary assets at sea are stored in a geospatial database and branch off into statistical models. The user sets a 'bloom indicator threshold' and is notified when it is exceeded. Vehicles passing through a bloom patch send real-time data to the ODSS verifying the location of the bloom patch based on indicators such as chlorophyll concentration. If the bloom is valid, its region of interest can be annotated and correlated with satellite data and in situ measurements like water samples within the same region. Using these sparse vehicle data, and remote sensing images from a prior day indicating a bloom could be advecting into the bay, the user can narrow down the sampling targets and locations to position robotic assets in the next 24-hour experiment cycle. Fig. 2 shows a conceptual view of how the ML capability fits into the ODSS.

\section{MACHINE LEARNing FOR ClassificAtion}

\section{A. Classifiers}

The classifiers examined in our problem were the Gaussian Naive Bayes (GNB) [20], and the Support 


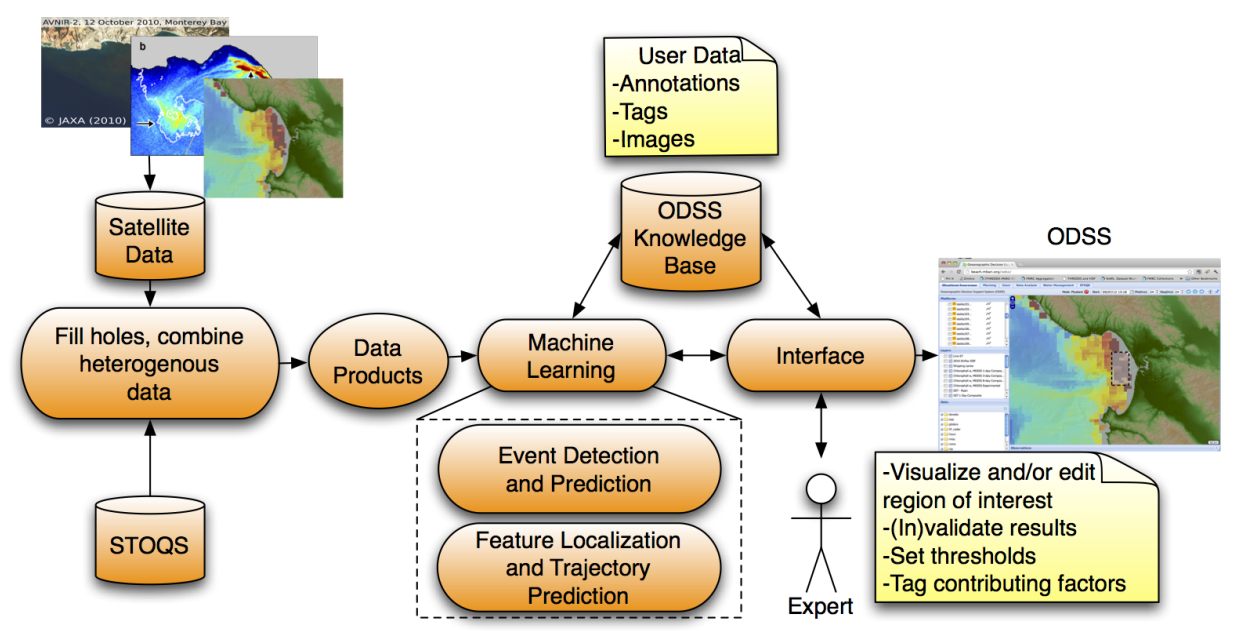

Fig. 2: A conceptual overview of how machine learning and event detection and response would work for a bloom event. AUVs could be retargeted based on expert assessment after being alerted about high chlorophyll levels based on ML models.

Vector Machine (SVM) [21]. A pair of classifiers were tested, with the goal to build a modular system applicable to different classification problems. Because of its sophisticated boundary definition, the SVM classifier provides significantly better results than the GNB, particularly in cases of unbalanced classes (such as this case). However, since GNB requires very little computation, it provides an effective means for quickly comparing the separability of the different classes using different feature sets, as well as a lower-bound estimate of the classification quality for different models. Since one crucial part of many classification tasks is determining the best set of feature vectors, this multistage approach using GNB first can be a useful time-saver. We use the Matlab package for libsvm [22], which provides efficient methods for training and testing SVMs. Also, 5-fold cross validation [21] was used to avoid overfitting.

\section{B. Classifier Quality}

There are various ways to judge the quality of a classifier when applied to a set of $M$ new observations. Most are based on the elements of the confusion matrix, being the numbers of True Positives (TP), True Negatives (TN), False Positives (FP), and False Negatives (FN). The accuracy is defined as $(\mathrm{TP}+\mathrm{TN}) / M$, while the precision is $\mathrm{TP} /(\mathrm{TP}+\mathrm{FP})$. In some cases, these metrics are relatively ineffective. For example, if there are fewer positive results than negative, then the accuracy is dominated by the true negatives and precision by false positives. Such a test case might look just as good under these metrics if the classifier were to identically map all new entries to the negative class. One measure which evenly balances performance between classes of different sizes is the Matthews Correlation Coefficient [23], given by:

$$
\mathrm{MCC}=\frac{\mathrm{TP} \times \mathrm{TN}-\mathrm{FP} \times \mathrm{FN}}{\sqrt{(\mathrm{TP}+\mathrm{FP})(\mathrm{TP}+\mathrm{FN})(\mathrm{TN}+\mathrm{FP})(\mathrm{TN}+\mathrm{FN})}} .
$$

An MCC of +1 represents perfectly correct prediction, -1 represents perfectly wrong prediction and 0 represents no better than random guessing. Examples of studies using MCC to assess the quality of binary classifiers can be found in the bioinformatics literature (e.g., [24], where MCC values range from 0.1 to 0.6 ).

\section{DATA ANALYSIS}

\section{A. On Ocean Satellite Data and Sources}

Satellite ocean color observation has seen a number of recent advances in atmospheric correction and the extraction of pigment concentrations [25], [26]. Both the Medium Resolution Imaging Spectrometer (MERIS) and Moderate Resolution Imaging Spectro-radiometer (MODIS) were instrumented with phytoplankton fluorescence ocean color bands, but only MERIS included a sensing band at $709 \mathrm{~nm}$, where red tide blooms cause a peak in the ocean surface reflectance. The Maximum Chlorophyll Index (MCI) derived from the MERIS instrument thus makes an excellent indicator for the extreme blooms common in the Monterey Bay [8]. However, contact was lost with Envisat, the satellite which carried the MERIS instrument, on April $8^{\text {th }}, 2012$, presenting an interesting opportunity to train on recent historical data to predict the now-unavailable MCI, and thus act as an extreme bloom detector.

\section{B. Analysis of Satellite Data}

We collected approximately 223 days of imagery where data existed from both MERIS and MODIS satellites between October 2010 and April 2012. Fig. 3 shows an example MCI reading, with bloom-like events occurring in Monterey Bay. Since MODIS and MERIS imagers have different sets of band frequencies, no direct method to correlate data between the two exists, leading to the question of whether an estimation can be made. The first step in this process was to spatially and temporally align the data, which also involved downsampling the $0.0754 \mathrm{~km}^{2}$-per-pixel MERIS data to the $1 \mathrm{~km}^{2}$-per-pixel resolution of the MODIS data. 


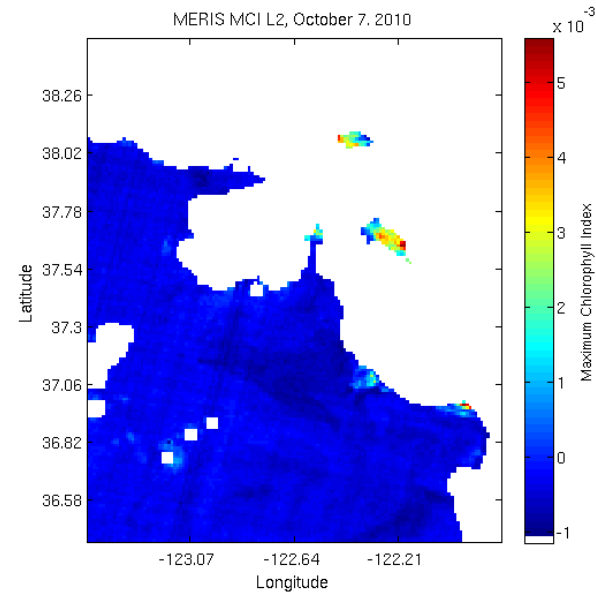

Fig. 3: Bloom-like events occurring in the northern portion of Monterey Bay and in San Francisco Bay on October $7^{\text {th }}$, 2010. White patches indicate areas of unknown data.

In order to incorporate spatial information into the classification model, we also considered various spatial filters. This has the effect of quickly increasing the length of the input feature vector, and thus the complexity of the model building process. To counteract this effect we use a fast, low-accuracy GNB classifier to quickly narrow the field of useful inputs and a slower, more accurate SVM to generate the final model.

\section{Spatial Information}

A simple ML approach treats each pixel independently in space and time, which can be computationally expensive. Neighboring pixels could be considered as features; however, this increases the dimensionality of the model exponentially. Using filters, spatial information can be encoded without significantly increasing the dimensionality of the data. These filters can also be chosen to respond to specific features, such as gradient, mean, edges, corners, frequencies, etc. [27] In this paper, we hypothesize that a high gradient across pixels of a given feature would potentially be more indicative of a bloom than the actual values of the same feature. Since remote sensing data can be patchy due to atmospheric effects, attempting to calculate relatively large features such as a gradient across a large number of pixels becomes increasingly difficult. Consequently, the data were interpolated up to the filter size using nearestneighbor interpolation [28], such that any calculated feature contains at least one known and non-interpolated value. This patchiness also led to some mismatch in the support set across data sources. Once we had chosen the set of all features to consider, we restricted the training to the intersection of those support sets so that each feature was represented in each pixel. This yielded 896,413 observed pixels over the 19-month period. Of these, 2, 868 (approximately $0.3 \%$ ) corresponded to extreme blooms based on the MCI threshold of $10^{-2.5}$. This extreme imbalance in classes prompted use of the
MCC as an indication of effectiveness, rather than other metrics like precision or accuracy.

\section{Data Sampling}

The statistical rarity of the minority class (i.e., bloom pixels) leads to poor results when training and testing sets are randomly assigned. For example, random sampling for cross-validation may yield subsets (called folds) containing too few samples of the minority class to build an effective predictive model. Instead, the data were stratifically randomly split, each fold containing the same ratio of classes as the whole data set.

Next, different data sampling methods were tested for further model refinements. First, undersampling of the majority class was examined, in which only a randomly selected portion of the samples in the majority class was used for training. However, the very high imbalance of classes meant that purely random undersampling provided little improvement.

The final sampling method tested was a selective undersampling, whereby the majority samples were sorted according to 'distance' from the closest members of the minority class. The distance metric used in this case was difference as measured in the feature space, based upon the idea that the majority samples most important for a decision boundary were those 'close' to the minority class. However, if one only used those samples closest to the minority class, overfitting was a significant problem. Hence, a random sample of majority class members below a certain distance percentile was chosen. Different sample sizes and percentiles were examined, and this method gave the best overall results.

\section{EXPERIMENTAL RESUlts}

MODIS data contain multiple features as potential inputs, but chlorophyll-A ( $\left.\mathrm{chl}_{A}\right)$ and fluorescence line height (flh) were chosen owing to their prior usage in bloom detection ([9], [11]). First tests involved using $\mathrm{chl}_{A}$ and flh individually. Adding new features quickly resulted in a sparsity problem, however, as each new feature introduced new undefined pixels and thus reduced the size of the working data set. One solution to this problem was to test individual features on the most restrictive subset, e.g., test with $\mathrm{chl}_{A}$ as the input feature but only testing on pixels where $\mathrm{chl}_{A}$ and flh are both known. Adding in filtered versions of both these features did not change the data set, due to the filtering implementation described above. The GNB classifier was used primarily for speed.

Preliminary results are summarized in Table I. First, among this data subset, $\mathrm{chl}_{A}$ did better than flh. This is curious, since prior work suggests flh is a better predictor than $\mathrm{chl}_{A}[9]$. However, these results vary depending upon different processes used to create the model, such as the sampling parameters used above.

After examining a number of spatial filters, the filter with the best performance was a blur filter, the result of averaging over a $5 \times 5$ spatial grid. We use $\mathrm{Chl}_{A}$ and 

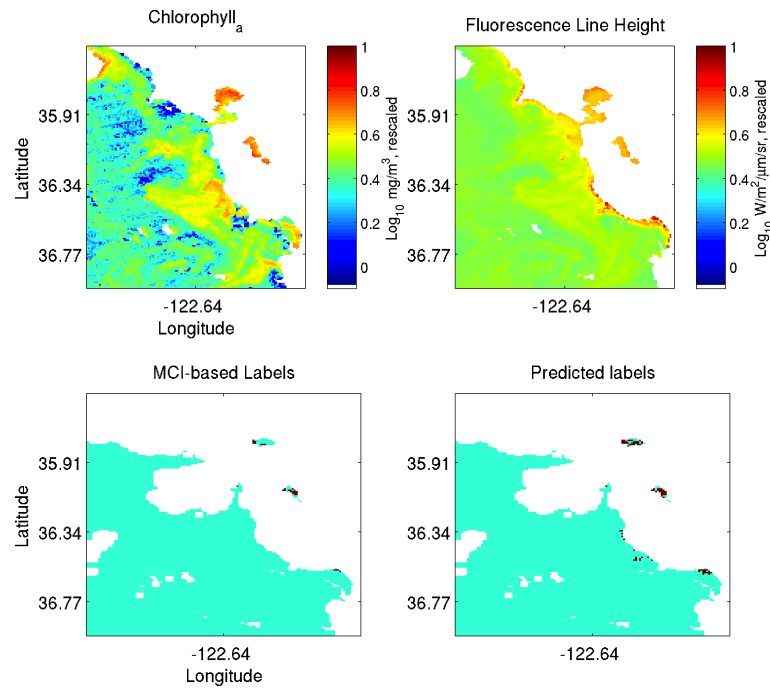

Fig. 4: Test results for Oct 7,2010 . The top row shows MODISmeasured $\operatorname{chl}_{A}$ and flh. The bottom row shows labels as calculated from MERIS MCI (left) and predicted by the GNB model (right). Bloom pixels are shown in red, and non-bloom pixels in blue. White areas represent unknown pixels either due to atmospheric interference or land. Since the labels are generated only for the subset of known MERIS and MODIS imagery, the labels are a smaller set of pixels than the MODIS images alone.

$\mathrm{flh}$ to denote the averaged versions of $\mathrm{chl}_{A}$ and flh, respectively. Using the individual filtered features resulted in an improvement of the MCC, likely because of the encoding of spatial information. It is interesting to note that in terms of MCC very little information seems to be lost by the spatial blurring. That is, e.g., using both $\mathrm{flh}$ and flh is not significantly better than $\mathrm{flh}$ alone. This is in agreement with the idea that blooms are a spatial phenomenon, and that the relative information between pixels is significant.

The best MODIS features explored so far are spatially-filtered versions of $\mathrm{chl}_{A}$ and flh, giving a lowerbound estimate of the MCC of 0.210 using the GNB classifier. This MCC corresponds to 2622 true positives, 845354 true negatives, 246 false negatives and 2680 false positives. Fig. 4 shows a typical result from this model. In this figure, one can see that there was a moderate number of false positives, while the model still detected the MCI-indicated bloom regions.

Due to the high imbalance of classes, since the MCC balances the error across classes, it is not surprising that there are much higher numbers of false positives than false negatives. This model achieves relatively high MCC performance by limiting false negatives, which is equally important in our application due to the cost of deploying field robots.

Next, a number of the false positives appear to be indicative of non-extreme blooms. For example, in Fig. 4, points along the coast show high fluorescence and chlorophyll readings, while not necessarily having high MCI. Since MCI works best on extreme blooms, it is quite possible that it is missing less extreme blooms

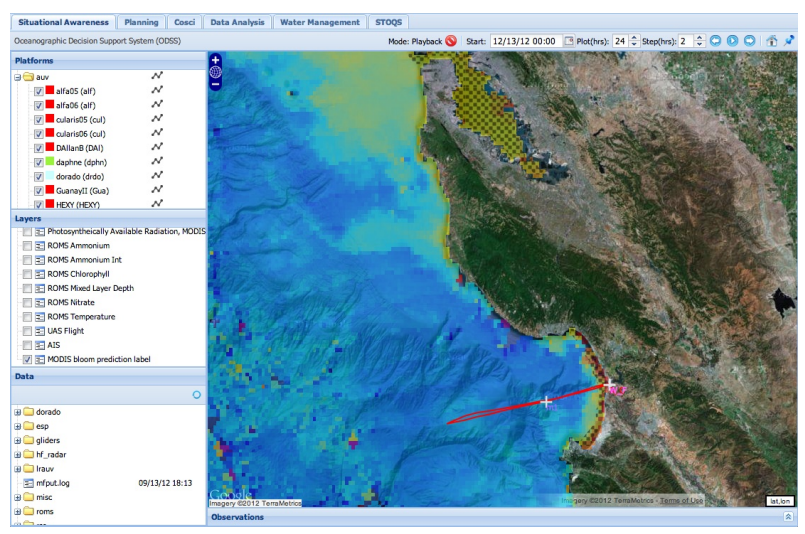

Fig. 5: Results of one model with the ODSS, showing predicted blooms as a checkerboard pattern superimposed upon a 3-day MODIS $\mathrm{chl}_{A}$ composite in the top-center of the map. The red line and white cross represent ship and buoy assets, respectively.

that are shown in relatively high readings of $\mathrm{chl}_{A}$ and flh.

Using a cross-validated grid-search for parameter selection, an SVM trained on $\mathrm{chl}_{A}, \mathrm{Chl}_{A}$, flh, flh was able to achieve an MCC of 0.428 . This primarily corresponded to a significant decrease in false positives, likely due to a more complex and better-fitting decision boundary. The integration of one model into the ODSS is shown in Fig. 5.

\section{CONCLUSIONS AND FUTURE WORK}

Our Machine Learning capability for extreme bloom detection is a step towards the more ambitious goal of a general event detection toolbox for robotic sampling in the coastal ocean. In the context of CANON field experiments and associated infrastructure including the ODSS, we aim to lay the groundwork for experiments tightly coupling ML inference and robotic exploration with AUVs.

We have gathered qualitative assessments from oceanographers from a March 2013 field experiment in the Southern California Bight area with the use of our toolbox to predict the extent of spreading of a bloom driven by a Deep Chlorophyl Maximum (DCM) when it enters the (visible) surface. We are currently analyzing the results for future work.

Our near-term future work is in three related directions: investigating methods of filling in sparse data such as spatial or space-time estimation, design of tailor-made classifiers that do not require all input features to be known a priori, and the design and implementation of cascades of simple classifiers. Doing so will build on this work in the context of more complex CANON field experiments. We also plan to expand the application domain beyond bloom detection to other oceanographic phenomena and features of interest in the Monterey Bay and to incorporate expert labels in our ML efforts. Such an investigation will necessarily include online as well as unsupervised learning methods for in situ feature detection on our AUV platforms. 
TABLE I: MCC results based on classification algorithm and input feature set. Here $c h l_{A}$ and $f l h$ denote the spatially averaged features.

\begin{tabular}{|c|c|c|c|c|c|c|c|}
\hline Algorithm & Feature Set & MCC & Accuracy & True Pos. & True Neg. & False Pos. & False Neg. \\
\hline GNB & flh & 0.116 & 0.852 & 2557 & 762474 & 132401 & 351 \\
\hline GNB & $\mathrm{chl}_{A}$ & 0.174 & 0.919 & 2754 & 863836 & 76719 & 115 \\
\hline GNB & $\mathrm{chl}_{A}, \mathrm{fh}$ & 0.210 & 0.946 & 2621 & 845414 & 48131 & 247 \\
\hline GNB & $\mathrm{flh}$ & 0.131 & 0.894 & 2401 & 799837 & 95038 & 507 \\
\hline GNB & $\mathrm{ch} l_{A}$ & 0.174 & 0.920 & 2735 & 864737 & 75817 & 134 \\
\hline GNB & $\mathrm{chl}_{A}, \mathrm{flh}$ & 0.210 & 0.946 & 2622 & 845354 & 48191 & 246 \\
\hline GNB & $\mathrm{chl}_{A}, \mathrm{ch} l_{A}, \mathrm{flh}, \mathrm{flh}$ & 0.212 & 0.946 & 2649 & 844986 & 48559 & 219 \\
\hline SVM & $\mathrm{chl}_{A}, \mathrm{ch} l_{A}, \mathrm{flh}, \mathrm{flh}$ & 0.428 & 0.997 & 1137 & 892237 & 1308 & 1731 \\
\hline
\end{tabular}

\section{ACKNOWLEDGMENT}

This work was supported in part by the National Science Foundation under grant No. 1124975 (Bernstein, Dolan, Graham, Rajan) and NOAA grant No. NA11NOS4780055 (Rajan). MBARI authors are also funded by a block grant from the David and Lucile Packard Foundation to MBARI.

\section{REFERENCES}

[1] P. Hoagland and S. Scatasta, "The economic effects of harmful algal blooms," in Ecology of Harmful Algae. Studies Series, E. Graneli and J. Turner, Eds. Springer-Verlag, 2006, ch. 30.

[2] K. Gomes, D. Cline, D. Edgington, M. Godin, T. Maughan, M. McCann, T. O'Reilly, F. Bahr, F. Chavez, M. Messi, J. Das, and K. Rajan, "ODSS: A Decision Support System for Ocean Exploration," in Workshop on Data-Driven Decision Guidance and Support Systems (DGSS), 29th IEEE International Conference on Data Engineering, Brisbane, Australia, 2013.

[3] J. Das, T. Maughan, M. McCann, M. Godin, T. OReilly, M. Messie, F. Bahr, K. Gomes, F. Py, J. Bellingham, G. S Sukhatme, and K. Rajan, "Towards Mixed-initiative, Multi-robot Field Experiments: Design, Deployment, and Lessons Learned," in Intelligent Robots and Systems (IROS), Jul. 2011.

[4] J. Ryan, M. McManus, R. Kudela, M. L. Artigas, J. Bellingham, F. Chavez, G. Doucette, D. Foley, M. Godin, J. Harvey, R. M. III, M. Messi, C. Mikulski, T. Pennington, F. Py, K. Rajan, I. Shulman, Z. Wang, and Y. Zhang, "Boundary influences on $\{\mathrm{HAB}\}$ phytoplankton ecology in a stratification-enhanced upwelling shadow," Deep Sea Research Part II: Topical Studies in Oceanography, no. 0, pp. -, 2013. [Online]. Available: http:// www.sciencedirect.com/science/article/pii/S0967064513000362

[5] J. P. Ryan, S. Johnson, A. Sherman, K. Rajan, F. Py, H. Thomas, J. Harvey, L. Bird, J. Paduan, and R. Vrijenhoek, "Mobile autonomous process sampling within coastal ocean observing systems," Limnology \& Oceanograhy: Methods, vol. 8, pp. 394402, 2010

[6] A. Garcia-Olaya, F. Py, J. Das, and K. Rajan, "An Online UtilityBased Approach for Sampling Dynamic Ocean Fields," IEEE Journal of Oceanic Engineering, vol. 37, no. 2, pp. 185-203, April 2012.

[7] K. Rajan, F. Py, and J. Berreiro, "Towards Deliberative Control in Marine Robotics," in Autonomy in Marine Robots, M. Seto, Ed. Springer Verlag, 2012, accepted: to be published Fall 2012.

[8] J. P. Ryan, H. M. Dierssen, R. M. Kudela, C. A. Scholin, K. S. Johnson, J. M. Sullivan, A. M. Fischer, E. V. Rienecker, P. R. McEnaney, and F. P. Chavez, "Coastal Ocean Physics Red Tides," Oceanography, vol. 18, no. 2, pp. 1-10, May 2005.

[9] J. P. Ryan, A. M. Fischer, R. M. Kudela, J. F. R. Gower, S. A. King, R. Marin III, and F. P. Chavez, "Influences of upwelling and downwelling winds on red tide bloom dynamics in Monterey Bay, California," Continental Shelf Research, vol. 29, no. 5-6, pp. 785-795, Mar. 2009.

[10] J. Das, K. Rajan, S. Frolov, F. Py, J. P. Ryan, D. A. Caron, and G. S. Sukhatme, "Towards Marine Bloom Trajectory Prediction for AUV Mission Planning," in Intl. conference on robotics and automation (ICRA), Feb. 2010.

[11] J. I. Allen, T. J. Smyth, J. R. Siddorn, and M. Holt, "How well can we forecast high biomass algal bloom events in a eutrophic coastal sea?" Harmful Algae, vol. 8, no. 1, pp. 70-76, Dec. 2008.
[12] O. Chapelle, P. Haffner, and V. Vapnik, "SVMs for HistogramBased Image Classification," IEEE Transactions on neural networks, pp. 1-11, May 1999

[13] P. I. Miller, J. D. Shutler, G. F. Moore, and S. B. Groom, "SeaWiFS discrimination of harmful algal bloom evolution," International Journal of Remote Sensing, vol. 27, no. 11, pp. 2287-2301, Jun. 2006

[14] M. C. Tomlinson, R. P. Stumpf, V. Ransibrahmanakul, E. W. Truby, G. J. Kirkpatrick, B. A. Pederson, G. A. Vargo, and C. A. Heil, "Evaluation of the use of SeaWiFS imagery for detecting Karenia brevis harmful algal blooms in the eastern Gulf of Mexico," Remote Sensing of Environment, vol. 91, no. 3-4, pp. 293-303, june 2004.

[15] R. Matarrese, A. Morea, K. Tijani, V. De Pasquale, M. Chiaradia, and G. Pasquariello, "A Specialized Support Vector Machine For Coastal Water Chlorophyll Retrieval From Water Leaving Reflectances." in Geoscience and Remote Sensing Symposium, Nov. 2008, pp. 1-4.

[16] D. W. Casbeer, S.-M. Li, R. W. Beard, R. K. Mehra, and T. W. McLain, "Forest fire monitoring with multiple small UAVs," in Proceedings of the 2005, American Control Conference, 2005. IEEE, 2005, pp. 3530-3535.

[17] S. Chien, B. Cichy, A. Davies, D. Tran, G. Rabideau, R. Castano, R. Sherwood, D. Mandl, S. Frye, S. Shulman et al., "An Autonomous Earth Observing Sensorweb," Intelligent Systems, IEEE, vol. 20, no. 3, pp. 16-24, 2005.

[18] N. M. Kakalis and Y. Ventikos, "Robotic Swarm Concept for Efficient Oil Spill Confrontation," Journal of hazardous materials, vol. 154, no. 1, pp. 880-887, 2008.

[19] M. Dunbabin and L. Marques, "Robotics for Environmental Monitoring," IEEE Robotics and Automation Magazine, vol. 19, no. 6, pp. 24-39, March 2012.

[20] C. Bustamante, L. Garrido, and R. Soto, "Comparing Fuzzy Naive Bayes and Gaussian Naive Bayes for Decision Making in RoboCup 3D," in MICAI 2006: Advances in Artificial Intelligence, A. Gelbukh and C. Reyes-Garcia, Eds. Springer Berlin / Heidelberg, 2006, pp. 237-247.

[21] C. M. Bishop, Pattern Recognition and Machine Learning (Information Science and Statistics). Secaucus, NJ, USA: SpringerVerlag New York, Inc., 2006.

[22] C.-C. Chang and C.-J. Lin, "'libsvm: a library for support vector machines"," Software available at http://www.csie.ntu.edu.tw/ cjlin/libsvm, 2001.

[23] P. Baldi, S. Brunak, Y. Chauvin, C. A. F. Andersen, and H. Nielsen, "Assessing the accuracy of prediction algorithms for classification: an overview," Bioinformatics, vol. 16, no. 5, pp. 412-424, 2000.

[24] Z. R. Yang, R. Thomson, P. McNeil, and R. M. Esnouf, "RONN: the bio-basis function neural network technique applied to the detection of natively disordered regions in proteins," Bioinformatics, vol. 21, no. 16, pp. 3369-3376, Aug. 2005.

[25] R. P. Stumpf and M. C. Tomplinson, "Remote Sensing Of Harmful Algal Blooms," in Remote Sensing of Coastal Aquatic Environments. US Government, Feb. 2005, pp. 277-296.

[26] C. R. McClain, "A Decade of Satellite Ocean Color Observations*," Annual Review of Marine Science, vol. 1, no. 1, pp. 19-42, Jan. 2009.

[27] D. A. Forsyth and J. Ponce, Computer Vision: A Modern Approach. Prentice Hall Professional Technical Reference, 2002.

[28] T. M. Lehmann, C. Gnner, and K. Spitzer, "Survey: Interpolation methods in medical image processing," IEEE Transactions on Medical Imaging, vol. 18, pp. 1049-1075, 1999. 